https://doi.org/10.15407/ujpe66.3.185

T. ABEBE ${ }^{1}$ CH. GASHU ${ }^{2}$ E. MOSISA ${ }^{2}$

${ }^{1}$ Department of Physics, Adama Science and Technology University

(P.O. Box 1888, Adama, Ethiopia; e-mail: tam1704@gmail.com)

2 Department of Physics, Jimma University

(P.O. Box 378, Jimma, Ethiopia)

\title{
GENERATION OF BRIGHT AND ENTANGLED \\ LIGHT FROM A NONDEGENERATE THREE-LEVEL LASER WITH PARAMETRIC AMPLIFIER AND COUPLED TO THERMAL RESERVOIR
}

\begin{abstract}
The detailed analysis of the two-mode quadrature squeezing and statistical properties of light generated by a nondegenerate three-level laser which has a parametric amplifier and coupled with a thermal reservoir is executed. The combination of the master equation and the stochastic differential equation is presented to study the nonclassical features of the light generated by the quantum system. Moreover, with the aid the resulting solutions together with the correlation properties of noise operators, we calculated the quadrature squeezing, entanglement, and mean number of photon pairs of the cavity light. It is found that the external small-amplitude driving radiation induces a strong correlation between the top and bottom states of three-level atoms to produce a high degree of squeezing. Moreover, the presence of a parametric amplifier is found to enhance the degree of squeezing of the cavity light. We have also established that an increase in the mean thermal photon number appears to degrade the squeezing, but enhances the mean number of photon pairs of the cavity light.

Keywords: parametric amplifier, quadrature squeezing, entanglement, mean number of photon pairs.
\end{abstract}

\section{Introduction}

Three-level cascade lasers have received considerable interest in connection with its potential as a source of light with interesting nonclassical features [1-18]. The quantum properties of light, in this device, is attributed to the atomic coherence that can be induced either by preparing the atoms initially in a coherent superposition of the top and bottom levels [7-9], or the coupling of these levels by an external radiation [10-12], or using these mechanisms together [13].

The parametric amplifier is a nonlinear crystal that involves three different modes of the radiation field:

(C) T. ABEBE, CH. GASHU, E. MOSISA, 2021

ISSN 2071-0194. Ukr. J. Phys. 2021. Vol. 66, No. 3 the signal, the idler, and the pump which are coupled by a nonlinear medium. In this device, a pump photon interacts with a nonlinear crystal inside a cavity and is down converted into two highly correlated photons of different frequencies [15-17]. Some authors have also studied quantum properties of light generated by a three-level laser whose cavity contains a parametric amplifier $[3,14,15,18-20]$. These works have indicated that the cavity radiation is found to be in a squeezed and entangled states under certain conditions. In addition, the mean and variance of the photon number for degenerate $[18,19]$ and nondegenerate [3, 20-23] three-level cascade lasers whose cavities contain a parametric amplifier have been determined in various cases. 
In most of the previous analyses, the laser cavity is coupled to a vacuum environment in which the effect of an external noise on the quantum properties and photon statistics is completely neglected. For instance, a squeezing of $93 \%$ has been realized in a degenerate three-level laser which has a parametric amplifier and is coupled to the vacuum reservoir [18]. Moreover, Abebe [21] has studied a nondegenerate three-level laser coupled to the vacuum reservoir in the presence of a parametric amplifier and classical pumping radiation. However, it is quite challenging for a real physical situations to be free from the effect of an external environment which unavoidably decouple the atomic correlations responsible for the quantum and statistical properties [21-23]. In this regard, Tesfa [23] has considered the effect of thermal noise on the squeezing, entanglement, and photon statistics of the cavity radiation generated by a correlated emission laser in the absence of a parametric amplifier and external pumping radiation that couple the top and bottom levels of a three-level atom. He has found that the thermal noise entering the cavity degrades the squeezing and entanglement, but enhances the mean number of photon pairs of the cavity light. On the other hand, the squeezing, entanglement, and statistical properties of the cavity radiation get enhanced with the introduction of a parametric amplifier. It is with this motivation that we will investigate these nonclassical properties for a radiation generated by a nondegenerate three-level laser whose cavity contains a nondegenerate parametric amplifier and is coupled to a two-mode thermal reservoir.

In this study, we analyze the squeezing, entanglement properties, and photon statistics of a twomode cavity light produced by a nondegenerate threelevel laser with a nondegenerate parametric amplifier which is coupled to a two-mode thermal reservoir via a single port mirror. In order to carry out our analyses, we first derive the master equation in the good cavity limit, linear and adiabatic approximations following the standard method in [15]. Employing the master equation and the stochastic differential equations, the solutions for $c$-number cavity mode variables, and correlation property of the noise forces associated with the normal ordering are determined. Using the resulting solutions, the mean number of photon pairs, quadrature fluctuations, and EPR-type operators of the cavity radiation were obtained in[24]. We investigate the effects of the para- metric amplifier and thermal noise on the squeezing, entanglement, and mean photon number of the cavity radiation. Moreover, based on the criterion for a continuous variable entanglement developed by Duan et al. [25], the relation between the squeezing and entanglement has been established.

The paper is organized as follows: in the first section, the Hamiltonian and the model are presented, and the master equation describing the dynamics of the optical device is derived. Making use of the master equation and stochastic differential equations, the solutions of the cavity mode variables are determined in the second section. Employing the solutions of the cavity mode variables, the mean photon-number and quadrature fluctuations of the two-mode cavity radiation are determined in section three. The entanglement quantification and mean number of photon pairs are analyzed for different cases in the last two sections.

\section{Hamiltonian and Master Equation}

The three-level cascade atoms initially prepared in a coherent superposition of the top and bottom levels are injected into the cavity at a constant rate, and removed from the laser cavity after some time, which is long enough for the atom to decay to levels other than the intermediate and bottom levels. Moreover, the top and bottom levels are coupled by the external driving radiation after being injected into the cavity. We consider the interaction of the three-level atoms with a resonant cavity mode and a nondegenerate parametric amplifier, as well as the damping of the cavity light by a thermal reservoir. As is clearly indicated, the top, intermediate, and bottom levels of a three-level atom are represented by $|l\rangle,|m\rangle$, and $|n\rangle$. We assume that the transitions between levels $|l\rangle$ and $|m\rangle$, and between levels $|m\rangle$ and $|n\rangle$ to be dipoleallowed, with direct transitions between levels $|l\rangle$ and $|n\rangle$ to be dipole-forbidden. We consider the case for which the two cavity modes are at resonance with the two transitions $|l\rangle \rightarrow|m\rangle$ and $|m\rangle \rightarrow|n\rangle$ having transition frequencies $\omega_{l m}$ and $\omega_{m n}$, respectively (Fig. 1).

In the nondegenerate three-level laser, a pump mode photon with frequency $\omega=\omega_{l m}+\omega_{m n}$ directly interacts with the nondegenerate parametric amplifier (NDPA) to produce the signal-idler photon pairs having the same frequencies as the two cavity modes [15-17]. The interaction of three-level atoms with a nondegenerate parametric amplifier can be

ISSN 2071-0194. Ukr. J. Phys. 2021. Vol. 66, No. 3 
described in the interaction picture, parametric approximation, and rotating-wave approximation by the quantum Hamiltonian

$\hat{H}_{1}=i \varepsilon\left(\hat{a}^{\dagger} \hat{b}^{\dagger}-\hat{a} \hat{b}\right)$,

in which $\varepsilon$ is considered to be real and constant, is proportional to the amplitude of the pump mode that drives the nonlinear crystal (NLC), and $\hat{a}$ and $\hat{b}$ are the annihilation operators for the two cavity modes. The master equation associated with this Hamiltonian has the form

$\frac{d}{d t} \hat{\rho}=\varepsilon\left(\hat{a}^{\dagger} \hat{b}^{\dagger} \hat{\rho}-\hat{\rho} a^{\dagger} \hat{b}^{\dagger}-\hat{a} \hat{b} \hat{\rho}+\hat{\rho} \hat{a} \hat{b}\right)$.

Moreover, the interaction of a three-level atom with the two cavity modes can be described by the Hamiltonian

$\hat{H}_{2}=i g\left[\hat{a}^{\dagger}|m\rangle\left\langle l\left|+\hat{b}^{\dagger}\right| n\right\rangle\langle m|-\hat{a}| l\rangle\langle m|-\hat{b}| m\rangle\langle n|\right]$,

where $g$ is the atom-cavity mode coupling constant assumed to be the same for both transitions. In this paper, we take the initial state of a single three-level atom to be

$|\psi(0)\rangle=A_{l}|l\rangle+A_{n}|n\rangle$,

where $A_{l}=\langle l \mid \psi(0)\rangle$ and $A_{n}=\langle n \mid \psi(0)\rangle$ are probability amplitudes for the atom to be in the top and bottom levels, respectively. It can be easily verified from Eq. (4) that the density matrix describing the initial state of the atom can be rewritten in the form

$\hat{\rho}^{a}(0)=\left(\begin{array}{ll}\rho_{1}(0) & \rho_{4}(0) \\ \rho_{3}(0) & \rho_{2}(0)\end{array}\right)$,

where $\rho_{1}(0)=\left|A_{l}\right|^{2}, \rho_{2}(0)=\left|A_{n}\right|^{2}, \rho_{3}(0)=\rho_{4}^{*}(0)=$ $=A_{l} A_{n}^{*}$. The off-diagonal terms in Eq. (5) are zero in a statistical mixture. On the other hand, in a coherent superposition of the top and bottom states of this three level laser, these terms are nonzero which leads to interesting physical properties that may be understood employing quantum mechanics.

The Hamiltonian describing the coupling of the upper and bottom levels by coherent radiation at resonance can be expressed as

$\hat{H}_{3}=i \frac{\Omega}{2}[|n\rangle\langle l|-| l\rangle\langle n|]$,

in which

$\Omega=2 \mu \beta_{0}$.

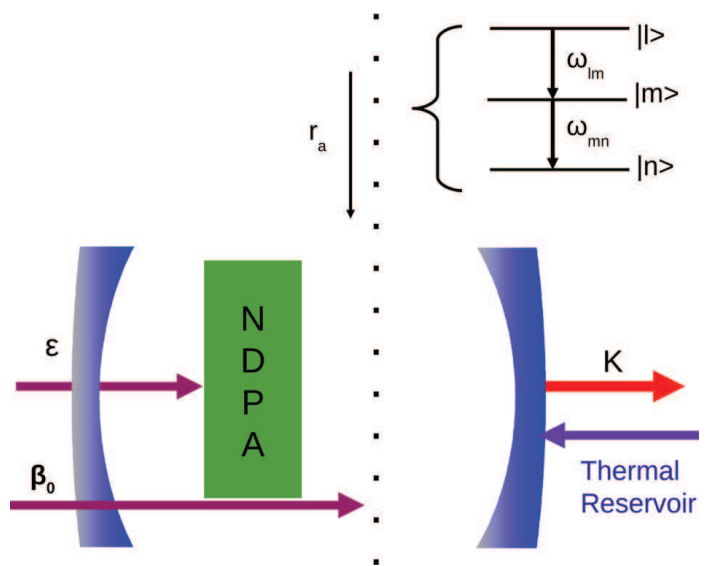

Fig. 1. Schematic representation of a nondegenerate threelevel laser that has a nondegenerate parametric amplifier and is coupled to the thermal reservoir

Here, $\beta_{0}$, considered to be real and constant, is proportional to the amplitude of the pump mode, and $\mu$ is the coupling constant between the pump mode and a three-level atom.

On the basis of Eqs. (3) and (6), the interaction of a three-level cascade atom, whose top and bottom levels are initially prepared in an arbitrary coherent superposition and also coupled by external coherent radiation, with a two-mode cavity radiation can be described in the interaction picture by the Hamiltonian

$\hat{H}^{\prime}=i g\left[\hat{a}^{\dagger}|m\rangle\langle l|-\hat{a}| l\rangle\left\langle m\left|+\hat{b}^{\dagger}\right| n\right\rangle\langle m|-\hat{b}| m\rangle\langle n|\right]+$

$+i \frac{\Omega}{2}[|n\rangle\langle l|-| l\rangle\langle n|]$.

In addition, we seek to consider when such atoms are injected into a cavity at constant rate $r_{a}$ and removed after some time $\tau$, which is long enough for the atoms to decay spontaneously to levels other than the middle or the lower level. The spontaneous decay rate $\gamma$ is taken to be the same for the two upper levels. In the good cavity limit, $\gamma \gg \kappa$, where $\kappa$ is the cavity damping constant, and the cavity mode variables change slowly compared with the atomic variables. Hence, the atomic variables will reac the steady state in a relatively short time. The time derivative of such variables can then be set to zero, while keeping the remaining terms at the time $t$. This procedure is referred as the adiabatic approximation scheme. Since the coupling constant is taken to be 
small, we confine ourselves to a linear analysis that amounts to dropping the terms of higher orders in $g$.

We thus find employing the linear and adiabatic approximation schemes in the good cavity limit that the equation of evolution of the density operator for the cavity modes has, in the absence of a damping through a coupled mirror, the form

$$
\begin{aligned}
& \frac{d \hat{\rho}}{d t}=\frac{A \Sigma_{1}}{2 \Delta}\left(2 \hat{a}^{\dagger} \hat{\rho} \hat{a}-\hat{a} \hat{a^{\dagger}} \hat{\rho}-\hat{\rho} \hat{a} \hat{a}^{\dagger}\right)+ \\
& +\frac{A \Sigma_{2}}{2 \Delta}\left(2 \hat{b} \hat{\rho} \hat{b}^{\dagger}-\hat{b}^{\dagger} \hat{b} \hat{\rho}-\hat{\rho} \hat{b}^{\dagger} \hat{b}\right)+ \\
& +\frac{A \Sigma_{3}}{2 \Delta}\left(\hat{a}^{\dagger} \hat{\rho} b^{\dagger}-\hat{\rho} \hat{b}^{\dagger} \hat{a}^{\dagger}+\hat{b} \hat{\rho} \hat{a}-\hat{a} \hat{\rho} \hat{b}\right)+ \\
& +\frac{A \Sigma_{4}}{2 \Delta}\left(\hat{a}^{\dagger} \hat{\rho} \hat{b}^{\dagger}-\hat{b}^{\dagger} \hat{a}^{\dagger} \hat{\rho}+\hat{b} \hat{\rho} \hat{a}-\hat{\rho} \hat{a} \hat{b}\right),
\end{aligned}
$$

where

$$
\begin{aligned}
& \Delta=\left(1+\zeta^{2}\right)\left(1+\frac{\zeta^{2}}{4}\right), \\
& \Sigma_{1}=\rho_{1}(0)\left(1+\frac{\zeta^{2}}{4}\right)+\rho_{2}(0) \frac{3 \zeta^{2}}{4}-\rho_{3}(0) \frac{3 \zeta}{2} \\
& \Sigma_{2}=\rho_{1}(0) \frac{3 \zeta^{2}}{4}+\rho_{2}(0)\left(1+\frac{\zeta^{2}}{4}\right)+\rho_{3}(0) \frac{3 \zeta}{2} \\
& \Sigma_{3}=-\rho_{1}(0) \frac{\zeta}{2}\left(1-\frac{\zeta^{2}}{2}\right)+\rho_{2}(0) \zeta\left(1+\frac{\zeta^{2}}{4}\right)- \\
& -\rho_{3}(0)\left(1-\frac{\zeta^{2}}{2}\right), \\
& \Sigma_{4}=-\rho_{1}(0) \zeta\left(1+\frac{\zeta^{2}}{4}\right)+\rho_{2}(0) \frac{\zeta}{2}\left(1-\frac{\zeta^{2}}{2}\right)- \\
& -\rho_{3}(0)\left(1-\frac{\zeta^{2}}{2}\right),
\end{aligned}
$$

with $\zeta=\Omega / \gamma, A=2 g^{2} r_{a} / \gamma^{2}$ is the linear gain coefficient, a parameter directly proportional to the rate (number of atoms per time) at which the atoms are injected into the cavity, for the three-level atom, and we have set $\rho_{3}(0)=\rho_{4}(0)$ for the mathematical rigor.

On the other hand, the time evolution of the density operator for a two-mode cavity radiation coupled to a two-mode thermal reservoir via a singleport mirror is found, following a procedure described by $[15,19]$, to be

$$
\begin{aligned}
& \frac{d \hat{\rho}}{d t}=-i\left[\hat{H}_{S}, \hat{\rho}(t)\right]+\frac{\kappa}{2} \bar{n}_{\mathrm{th}}\left(2 \hat{a}^{\dagger} \hat{\rho} \hat{a}-\hat{a} \hat{a}^{\dagger} \hat{\rho}-\hat{\rho} \hat{a} \hat{a}^{\dagger}\right)+ \\
& +\frac{\kappa}{2}\left(\bar{n}_{\mathrm{th}}+1\right)\left(2 \hat{a} \hat{\rho} \hat{a}^{\dagger}-\hat{a}^{\dagger} \hat{a} \hat{\rho}-\hat{\rho} \hat{a}^{\dagger} \hat{a}\right)+
\end{aligned}
$$

\section{8}

$$
\begin{aligned}
& +\frac{\kappa}{2}\left(\bar{n}_{\mathrm{th}}+1\right)\left(2 \hat{b} \hat{\rho} \hat{b}^{\dagger}-\hat{b}^{\dagger} \hat{b} \hat{\rho}-\hat{\rho} \hat{b}^{\dagger} \hat{b}\right)+ \\
& +\frac{\kappa}{2} \bar{n}_{\mathrm{th}}\left(2 \hat{b}^{\dagger} \hat{\rho} \hat{b}-\hat{b} \hat{b}^{\dagger} \rho-\hat{\rho} \hat{b} \hat{b}^{\dagger}\right),
\end{aligned}
$$

where we assume $\kappa_{a}=\kappa_{b}=\kappa$ is cavity damping constant.

Finally, on account of Eqs. (2), (9), and (11), the equation of evolution of the density operator for the cavity modes takes the form

$$
\begin{aligned}
& \frac{d \hat{\rho}}{d t}=\varepsilon\left[\hat{\rho} \hat{a} \hat{b}-\hat{a} \hat{b} \hat{\rho}+\hat{a}^{\dagger} \hat{b}^{\dagger} \hat{\rho}-\hat{\rho} \hat{a}^{\dagger} \hat{b}^{\dagger}\right]+ \\
& +\frac{\kappa}{2}\left(\bar{n}_{\mathrm{th}}+1\right)\left[2 \hat{a} \hat{\rho} \hat{a}^{\dagger}-\hat{a}^{\dagger} \hat{a} \hat{\rho}-\hat{\rho} \hat{a}^{\dagger} \hat{a}\right]+ \\
& +\frac{1}{2}\left(\frac{A \Sigma_{1}}{\Delta}+\kappa \bar{n}_{\mathrm{th}}\right)\left[2 \hat{a}^{\dagger} \hat{\rho} \hat{a}-\hat{a} \hat{a}^{\dagger} \hat{\rho}-\hat{\rho} \hat{a} \hat{a}^{\dagger}\right]+ \\
& +\frac{1}{2}\left(\frac{A \Sigma_{2}}{\Delta}+\kappa\left(\bar{n}_{\mathrm{th}}+1\right)\right)\left[2 \hat{b} \hat{\rho} \hat{b}^{\dagger}-\hat{b}^{\dagger} \hat{b} \hat{\rho}-\hat{\rho} \hat{b}^{\dagger} \hat{b}\right]+ \\
& +\frac{1}{2} \kappa \bar{n}_{\mathrm{th}}\left[2 \hat{b}^{\dagger} \hat{\rho} \hat{b}-\hat{b} \hat{b}^{\dagger} \rho-\hat{\rho} \hat{b} \hat{b}^{\dagger}\right]- \\
& -\frac{A \Sigma_{3}}{2 \Delta}\left[\hat{a} \hat{b} \hat{\rho}-\hat{a}^{\dagger} \hat{\rho} \hat{b}^{\dagger}+\hat{\rho} \hat{b}^{\dagger} \hat{a}^{\dagger}-\hat{b} \hat{\rho} \hat{a}\right]- \\
& -\frac{A \Sigma_{4}}{2 \Delta}\left[\hat{b}^{\dagger} \hat{a}^{\dagger} \hat{\rho}-\hat{a}^{\dagger} \hat{\rho} \hat{b}^{\dagger}+\hat{\rho} \hat{a} \hat{b}-\hat{b} \hat{\rho} \hat{a}\right] .
\end{aligned}
$$

This is the master equation for the cavity modes of a nondegenerate three-level laser whose cavity contains a nondegenerate parametric amplifier and is coupled to a two-mode thermal reservoir.

On the basis of Eqs. (A12) and (A13), we can write

$$
\begin{aligned}
& \frac{d}{d t} \alpha(t)=-\frac{\mu_{a}}{2} \alpha(t)+\frac{\nu_{a}}{2} \beta^{*}(t)+f_{\alpha}(t), \\
& \frac{d}{d t} \beta^{*}(t)=-\frac{\mu_{b}}{2} \beta^{*}(t)-\frac{\nu_{b}}{2} \alpha(t)+f_{\beta}^{*}(t) .
\end{aligned}
$$

where $f_{\alpha}(t)$ and $f_{\beta}^{*}(t)$ are noise forces whose properties remain to be determined.

We now proceed to determine the properties of the noise forces. It is obvious that the expectation values of Eqs. (13) and (14) are identical to Eqs. (A12) and (A13) provided that

$$
\left\langle f_{\alpha}(t)\right\rangle=\left\langle f_{\beta}(t)\right\rangle=0 \text {. }
$$

Moreover, making use of Eqs. (13) and (14), we can verify that

$$
\left\langle f_{\alpha}\left(t^{\prime}\right) f_{\alpha}(t)\right\rangle=\left\langle f_{\beta}(t) f_{\beta}\left(t^{\prime}\right)\right\rangle=0,
$$




$$
\begin{aligned}
& \left\langle f_{\alpha}^{*}(t) f_{\beta}\left(t^{\prime}\right)\right\rangle=\left\langle f_{\beta}^{*}\left(t^{\prime}\right) f_{\alpha}(t)\right\rangle=0, \\
& \left\langle f_{\alpha}\left(t^{\prime}\right) f_{\alpha}^{*}(t)\right\rangle=\left(\frac{A \Sigma_{1}}{\Delta}+\kappa \bar{n}_{\mathrm{th}}\right) \delta\left(t-t^{\prime}\right), \\
& \left\langle f_{\beta}\left(t^{\prime}\right) f_{\beta}^{*}(t)\right\rangle=\kappa \bar{n}_{\mathrm{th}} \delta\left(t-t^{\prime}\right), \\
& \left\langle f_{\beta}\left(t^{\prime}\right) f_{\alpha}(t)\right\rangle=-\frac{\nu_{b}}{2} \delta\left(t-t^{\prime}\right) .
\end{aligned}
$$

We note that Eqs. (15)-(20) represent the correlation properties of the noise forces $f_{\alpha}(t)$ and $f_{\beta}(t)$ associated with the normal ordering.

It proves to be more convenient to introduce a new parameter $x$ defined by

$\rho_{1}(0)=\frac{(1-x)}{2}$,

with $-1 \leq x \leq 1$. For three-level atoms initially in a coherent superposition of the top and bottom levels, we find with the use of Eq. (21) that

$$
\begin{aligned}
\rho_{2}(0) & =\frac{1+x}{2}, \\
\rho_{3}(0) & =\frac{\sqrt{1-x^{2}}}{2} .
\end{aligned}
$$

Hence, substituting Eqs. (15)-(20) along with (21)(23) into Eqs. (13) and (14), we obtain

$$
\begin{aligned}
& \frac{d}{d t} \alpha(t)=-\xi_{+} \alpha(t)-\eta_{+} \beta^{*}(t)+f_{\alpha}(t), \\
& \frac{d}{d t} \beta^{*}(t)=-\xi_{-} \beta^{*}(t)-\eta_{-} \alpha(t)+f_{\beta}^{*}(t),
\end{aligned}
$$

where

$\xi_{ \pm}=\frac{1}{2}\left(\kappa+\frac{A}{2 \Delta}\left[\frac{3 \zeta}{2} \sqrt{1-x^{2}}+x\left(1-\frac{\zeta^{2}}{2}\right) \mp\left(1+\zeta^{2}\right)\right]\right)$,

$\eta_{ \pm}=-\frac{1}{2}\left(2 \epsilon+\frac{A}{2 \Delta}\left[\frac{\zeta}{2}\left(1+\zeta^{2}\right) \pm\left[\frac{3 x \zeta}{2}-\right.\right.\right.$

$\left.\left.\left.-\sqrt{1-x^{2}}\left(1-\frac{\zeta^{2}}{2}\right)\right]\right]\right)$.

We find the solutions of the coupled differential equations (24) and (25) by using the procedure described in $[20,23]$ to be

$\alpha(t)=A_{+}(t) \alpha(0)+B_{+}(t) \beta^{*}(0)+F_{+}(t)+W_{+}(t)$,

ISSN 2071-0194. Ukr. J. Phys. 2021. Vol. 66, No. 3
$\beta(t)=A_{-}(t) \beta(0)+B_{-}(t) \alpha^{*}(0)+F_{-}(t)+W_{-}(t)$,

where

$A_{ \pm}(t)=\frac{1}{2}\left[(1 \pm p) e^{-\lambda_{-} t}+(1 \mp p) e^{-\lambda_{+} t}\right]$,

$B_{ \pm}(t)=\frac{q_{ \pm}}{2}\left[e^{-\lambda_{+} t}-e^{-\lambda_{-} t}\right]$

$F_{+}(t)=\frac{1}{2} \int_{0}^{t}\left[(1+p) e^{-\lambda_{-}\left(t-t^{\prime}\right)}+\right.$

$\left.+(1-p) e^{-\lambda_{+}\left(t-t^{\prime}\right)}\right] f_{\alpha}\left(t^{\prime}\right) d t^{\prime}$

$F_{-}(t)=\frac{1}{2} \int_{0}^{t}\left[(1-p) e^{-\lambda_{-}\left(t-t^{\prime}\right)}+\right.$

$\left.+(1+p) e^{-\lambda_{+}\left(t-t^{\prime}\right)}\right] f_{\beta}\left(t^{\prime}\right) d t^{\prime}$,

$W_{+}(t)=\frac{q_{+}}{2} \int_{0}^{t}\left[e^{-\lambda_{+}\left(t-t^{\prime}\right)}-e^{-\lambda_{-}\left(t-t^{\prime}\right)}\right] f_{\beta}^{*}\left(t^{\prime}\right) d t^{\prime}$,

$W_{-}(t)=\frac{q_{-}}{2} \int_{0}^{t}\left[e^{-\lambda_{+}\left(t-t^{\prime}\right)}-e^{-\lambda_{-}\left(t-t^{\prime}\right)}\right] f_{\alpha}^{*}\left(t^{\prime}\right) d t^{\prime}$,

and

$p=\frac{1+\zeta^{2}}{V}$,

$q_{ \pm}=\frac{-\left[\frac{\zeta}{2}\left(1+\zeta^{2}\right)+4 \varepsilon\left(\frac{\Delta}{A}\right)\right]}{V} \mp$

$\mp \frac{\left[\frac{3 x \zeta}{2}-\sqrt{1-x^{2}}\left(1-\frac{\zeta^{2}}{2}\right)\right]}{V}$,

$\lambda_{ \pm}=\frac{\kappa}{2}+\frac{A}{4 \Delta}\left[\frac{3 \zeta}{2} \sqrt{1-x^{2}}+x\left(1-\frac{\zeta^{2}}{2}\right) \pm V\right]$,

with

$V=\left[\left(1+\zeta^{2}\right)^{2}+\left(\frac{\zeta}{2}\left(1+\zeta^{2}\right)+\frac{4 \varepsilon \Delta}{A}\right)^{2}-\right.$

$\left.-\left(\frac{3 x \zeta}{2}-\sqrt{1-x^{2}}\left(1-\frac{\zeta^{2}}{2}\right)\right)^{2}\right]^{1 / 2}$.

\section{Quadrature Squeezing}

In this section, we will study the quadrature squeezing of the light produced by a nondegenerate threelevel laser with a nondegenerate parametric amplifier coupled to the thermal reservoir via a singleport mirror. In general, the squeezing properties of a two-mode cavity radiation can be described by two quadrature operators $[20,21]$

$\hat{c}_{+}=\left(\hat{c}^{\dagger}+\hat{c}\right)$, 
$\hat{c}_{-}=i\left(\hat{c}^{\dagger}-\hat{c}\right)$,

where

$\hat{c}=\frac{1}{\sqrt{2}}(\hat{a}+\hat{b})$,

where $\hat{a}$ and $\hat{b}$ represent the separate modes. In view of the commutation relation $\left[\hat{c}, \hat{c}^{\dagger}\right]=1$, the quadrature operators $\hat{c}_{+}$and $\hat{c}_{-}$are Hermitian and satisfy the commutation relation

$\left[\hat{c}_{+}, \hat{c}_{-}\right]=2 i$.

On the basis of these definitions, a two-mode light is said to be in a squeezed state, if either $\Delta c_{+}^{2}<1$ and $\Delta c_{-}^{2}>1$ or $\Delta c_{+}^{2}>1$ and $\Delta c_{-}^{2}<1$ such that $\Delta c_{+} \Delta c_{-} \geq 1$. The variances of the quadrature operators can be expressed as

$\Delta c_{ \pm}^{2}=\left\langle\hat{c}_{ \pm}^{2}\right\rangle-\left\langle\hat{c}_{ \pm}\right\rangle^{2}$.

It is possible to express the variance of the quadrature operators (40) and (41) in terms of the $c$-number variables associated with the normal ordering taking the cavity modes to be initially in a two-mode thermal state as

$\Delta c_{ \pm}^{2}=1+\left\langle\alpha^{*}(t) \alpha(t)\right\rangle+\left\langle\beta^{*}(t) \beta(t)\right\rangle+\left\langle\alpha^{*}(t) \beta(t)\right\rangle+$

$+\left\langle\alpha(t) \beta^{*}(t)\right\rangle \pm\left[\left\langle\alpha^{*}(t) \beta^{*}(t)\right\rangle+\langle\alpha(t) \beta(t)\rangle+\right.$

$\left.+\frac{1}{2}\left(\left\langle\alpha^{2}(t)\right\rangle+\left\langle\alpha^{* 2}(t)\right\rangle+\left\langle\beta^{* 2}(t)\right\rangle+\left\langle\beta^{2}(t)\right\rangle\right)\right]$.

In view of the fact that the noise force at the time $t$ does not affect the cavity mode variables at earlier times and taking the cavity modes to be initially in a vacuum state, it is also possible to verify at a steady state that

$$
\begin{aligned}
& \left\langle\alpha^{2}(t)\right\rangle=\left\langle\beta^{2}(t)\right\rangle=\left\langle\alpha^{*}(t) \beta(t)\right\rangle=0, \\
& \left\langle\alpha^{*}(t) \alpha(t)\right\rangle=\frac{\left(\frac{A \Sigma_{1}}{\Delta}+\kappa \bar{n}_{\mathrm{th}}\right)(1-p)^{2}+\kappa \bar{n}_{\mathrm{th}} q_{+}^{2}}{8 \lambda_{+}}+ \\
& +\frac{\left(\frac{A \Sigma_{1}}{\Delta}+\kappa \bar{n}_{\mathrm{th}}\right)(1+p)^{2}+\kappa \bar{n}_{\mathrm{th}} q_{+}^{2}}{8 \lambda_{-}}- \\
& -\frac{\left(\frac{A \Sigma_{4}}{\Delta}-2 \varepsilon\right) q_{+}(1-p)}{8 \lambda_{+}}+\frac{\left(\frac{A \Sigma_{4}}{\Delta}-2 \varepsilon\right) q_{+}(1+p)}{8 \lambda_{-}}+ \\
& +\frac{\left(\frac{A \Sigma_{1}}{\Delta}+\kappa \bar{n}_{\mathrm{th}}\right)\left(1-p^{2}\right)-\kappa \bar{n}_{\mathrm{th}} q_{+}^{2}}{2\left(\lambda_{+}+\lambda_{-}\right)}-
\end{aligned}
$$

$-\frac{\left(\frac{A \Sigma_{4}}{\Delta}-2 \varepsilon\right) q_{+} p}{2\left(\lambda_{+}+\lambda_{-}\right)}$

$\left\langle\beta^{*}(t) \beta(t)\right\rangle=\frac{\left(\frac{A \Sigma_{1}}{\Delta}+\kappa \bar{n}_{\mathrm{th}}\right) q_{-}^{2}+\kappa \bar{n}_{\mathrm{th}}(1+p)^{2}}{8 \lambda_{+}}+$

$+\frac{\left(\frac{A \Sigma_{1}}{\Delta}+\kappa \bar{n}_{\mathrm{th}}\right) q_{-}^{2}+\kappa \bar{n}_{\mathrm{th}}(1-p)^{2}}{8 \lambda_{-}}-$

$-\frac{\left(\frac{A \Sigma_{4}}{\Delta}-2 \varepsilon\right) q_{-}(1+p)}{8 \lambda_{+}}+\frac{\left(\frac{A \Sigma_{4}}{\Delta}-2 \varepsilon\right) q_{-}(1-p)}{8 \lambda_{-}}-$

$-\frac{\left(\frac{A \Sigma_{1}}{\Delta}+\kappa \bar{n}_{\mathrm{th}}\right) q_{-}^{2}-\kappa \bar{n}_{\mathrm{th}}\left(1-p^{2}\right)}{2\left(\lambda_{+}+\lambda_{-}\right)}+$

$+\frac{\left(\frac{A \Sigma_{4}}{\Delta}-2 \varepsilon\right) q_{-} p}{2\left(\lambda_{+}+\lambda_{-}\right)}$

$\langle\alpha(t) \beta(t)\rangle=$

$=\frac{\left(\frac{A \Sigma_{1}}{\Delta}+\kappa \bar{n}_{\mathrm{th}}\right) q_{-}(1-p)+\kappa \bar{n}_{\mathrm{th}} q_{+}(1+p)}{8 \lambda_{+}}-$

$-\frac{\left(\frac{A \Sigma_{1}}{\Delta}+\kappa \bar{n}_{\mathrm{th}}\right) q_{-}(1+p)+\kappa \bar{n}_{\mathrm{th}} q_{+}(1-p)}{8 \lambda_{-}}-$

$-\frac{\left(\left(\frac{A \Sigma_{4}}{2 \Delta}-\varepsilon\right)\left(1-p^{2}+q_{-} q_{+}\right)\right.}{8 \lambda_{+}}+$

$+\frac{\left(\left(\frac{A \Sigma_{4}}{2 \Delta}-\varepsilon\right)\left(1-p^{2}+q_{-} q_{+}\right)\right.}{8 \lambda_{-}}+$

$+\frac{\left(\frac{A \Sigma_{1}}{\Delta}+\kappa \bar{n}_{\mathrm{th}}\right) q_{-} p-\kappa \bar{n}_{\mathrm{th}} q_{+} p}{2\left(\lambda_{+}+\lambda_{-}\right)}-$

$-\frac{\left(\frac{A \Sigma_{4}}{2 \Delta}-\varepsilon\right)\left(1+p^{2}-q_{-} q_{+}\right)}{2\left(\lambda_{+}+\lambda_{-}\right)}$.

We observe that Eqs. (47) and (48), respectively, represent the steady state mean photon number of the cavity modes $a$ and $b$.

With the aid of Eqs. (46)-(49), Eq. (45) turns out to be

$\Delta c_{ \pm}^{2}=1+\left[\frac{A}{\Delta}(\Gamma \mp \chi)+2\left(\kappa \bar{n}_{\mathrm{th}} \pm \varepsilon\right)\right] \times$

$\times\left[\frac{\left(\lambda_{+}+\lambda_{-}\right)^{2}+4 \lambda_{+} \lambda_{-}}{8 \lambda_{+} \lambda_{-}\left(\lambda_{+}+\lambda_{-}\right)}\right]+$

$+\left[\left(\frac{A \Gamma}{\Delta}+\kappa \bar{n}_{\mathrm{th}}\right)\left(p^{2}+q_{-}^{2} \mp 2 q_{-} p\right)+\right.$

$+\kappa \bar{n}_{\mathrm{th}}\left(p^{2}+q_{+}^{2} \pm 2 q_{+} p\right)+\left(\frac{A \chi}{\Delta}-2 \varepsilon\right) \times$

$\left.\times\left[p\left(q_{+}-q_{-}\right) \pm\left(p^{2}-q_{-} q_{+}\right)\right]\right] \times$

$\times\left[\frac{\left(\lambda_{+}+\lambda_{-}\right)^{2}-4 \lambda_{+} \lambda_{-}}{8 \lambda_{+} \lambda_{-}\left(\lambda_{+}+\lambda_{-}\right)}\right]+$

ISSN 2071-0194. Ukr. J. Phys. 2021. Vol. 66, No. 3 


$$
\begin{aligned}
& +\left[\left(\frac{A \Gamma}{\Delta}+\kappa \bar{n}_{\mathrm{th}}\right)\left(p \mp q_{-}\right)-\kappa \bar{n}_{\mathrm{th}}\left(p \pm q_{+}\right)+\right. \\
& \left.+\left(\frac{A \chi}{2 \Delta}-\varepsilon\right)\left(q_{+}+q_{-}\right)\right]\left[\frac{\left(\lambda_{+}-\lambda_{-}\right)}{4 \lambda_{+} \lambda_{-}}\right]
\end{aligned}
$$

where

$\Gamma=\frac{1}{2}+\frac{\zeta^{2}}{2}+\frac{\zeta^{2} x}{4}-\frac{3 \zeta}{4} \sqrt{1-x^{2}}-\frac{x}{2}$,

$\chi=-\frac{\zeta}{4}\left(1+\zeta^{2}\right)+\frac{3 \zeta x}{4}-\frac{\sqrt{1-x^{2}}}{2}\left(1-\frac{\zeta^{2}}{2}\right)$.

Equation (50) represents the variances of the steady state of cavity modes for a nondegenerate three-level laser whose cavity contains a nondegenerate parametric amplifier and is coupled to a twomode thermal reservoir. At the steady state, the system and the environment assume thermal equilibrium with each other. We observe that the equations of evolution of $\alpha(t)$ and $\beta(t)$ do not have well-behaved solutions for $\lambda_{-}<0$. Hence, we note that the threshold condition for the system under consideration is attained, when

$\lambda_{-}=0$.

This condition yields

$\varepsilon_{\max }=\frac{A}{4 \Delta}\left[-\frac{\zeta\left(1+\zeta^{2}\right)}{2}+\tilde{\Delta}\right]$,

in which

$\tilde{\Delta}=\sqrt{U^{2}-\left(1+\zeta^{2}\right)^{2}+\left(\frac{3 x \zeta}{2}-\sqrt{1-x^{2}}\left(1-\frac{\zeta^{2}}{2}\right)\right)^{2}}$,

$U=\frac{2 k \Delta}{A}+\frac{3 \zeta}{2} \sqrt{1-x^{2}}+x\left(1-\frac{\zeta^{2}}{2}\right)$

Equation (54) provides the maximum possible value of the amplitude of a parametric amplifier for the fixed values of the other parameters. The analysis is therefore carried out for $\varepsilon \leq \varepsilon_{\max }$.

In order to investigate the dependence of the squeezing on the amplitude of external driving radiation, the initial preparation of three-level atoms, amplitude of a parametric amplifier, and linear gain coefficient, we plot the minus quadrature variance versus these parameters keeping some parameters fixed.

We clearly see from Fig. 2 that the two-mode cavity radiation exhibits the squeezing for all values of $\bar{n}_{\text {th }}$

ISSN 2071-0194. Ukr. J. Phys. 2021. Vol. 66, No. 3

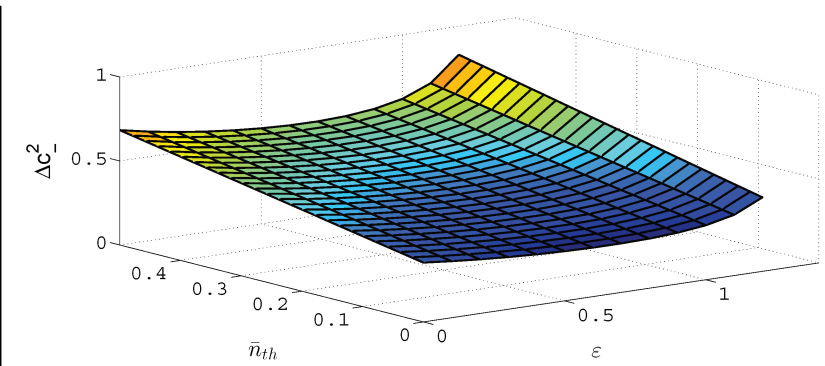

Fig. 2. A plot of the minus quadrature variance [Eq. (50)] of the two-mode cavity radiation at the steady state for $\zeta=0.001$, $x=0.1, \kappa=0.75$, and $A=100$

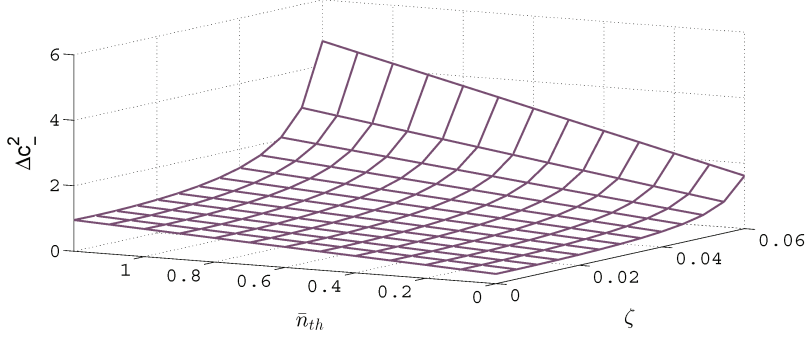

Fig. 3. A plot of the minus quadrature variance [Eq. (50)] of the two-mode cavity radiation at the steady state for $A=100$, $x=0.1, \kappa=0.75$, and $\varepsilon=0.5$

and $\varepsilon \leq 1.2$. Squeezing occurs for $\bar{n}_{\text {th }} \leq 0.5$ and $0 \leq$ $\leq \varepsilon \leq 1.2$ in this particular figure as the value of the minus quadrature variance is less than one. It is found that a maximum squeezing of $71 \%$ occurs for $\bar{n}_{\mathrm{th}}=$ 0 and $\varepsilon=0.7$. It is also verified that the degree of squeezing decreases with the mean photon number of the thermal reservoir for every values of the amplitude of a parametric amplifier.

It is clearly evident from Fig. 3 that the two-mode cavity radiation exhibits the squeezing for certain values of $\bar{n}_{\text {th }}$ and $\zeta$. It is not difficult to see that the degree of squeezing decreases with the mean photon number of the thermal reservoir. Furthermore, a maximum squeezing of $70 \%$ is found to occur for $\bar{n}_{\text {th }}=\zeta=0$. It is also possible to see that the squeezing does not occur for certain values $\bar{n}_{\text {th }}$ and $\zeta$. For instance, the squeezing does not occur for $\bar{n}_{\text {th }} \geq 0.7$ and $\zeta \geq 0.04$ in this case. This is an indication of the smallness of the system to be easily disturbed, when the large thermal reservoir's influence escalates (increment in the temperature and then mean photon number).

As we clearly see from Fig. 4, the squeezing decreases with the cavity damping constant for a specific value of the amplitude of a parametric ampli- 


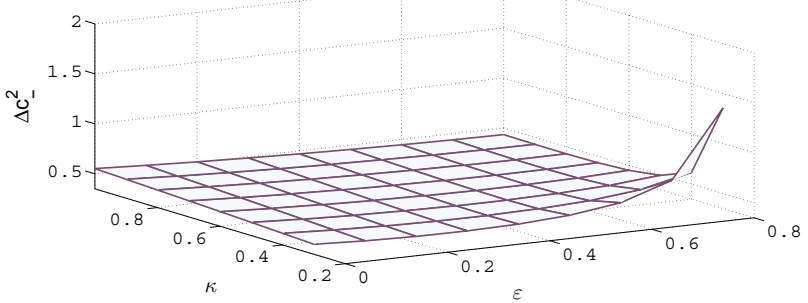

Fig. 4. A plot of the minus quadrature variance [Eq. (50)] of the two-mode cavity radiation at the steady state for $A=100$, $\bar{n}_{\mathrm{th}}=0, x=0.1$, and $\zeta=0.001$

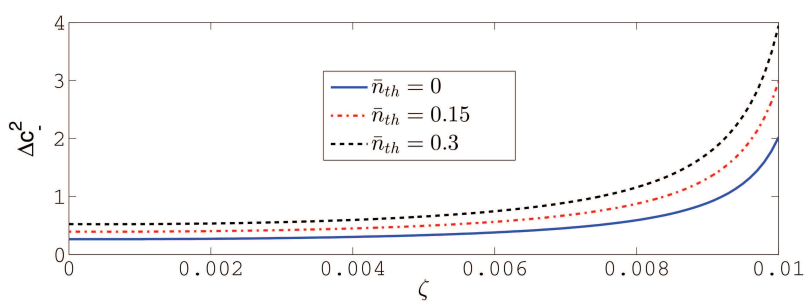

Fig. 5. Plots of the minus quadrature variance [Eq. (50)] of the two-mode cavity radiation at the steady state for $x=0.03$, $A=1000, \kappa=0.75, \varepsilon=0.5$, and for different values of $\bar{n}_{\mathrm{th}}$

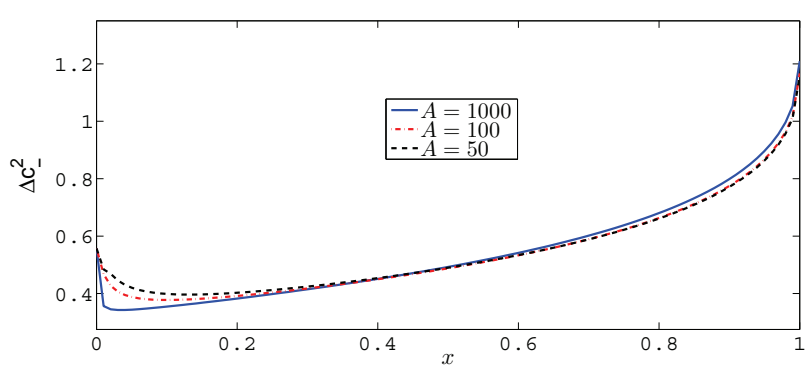

Fig. 6. Plots of the minus quadrature variance [Eq. (50)] of the two-mode cavity radiation at the steady state for $k=0.75$, $\varepsilon=0.5, \bar{n}_{\mathrm{th}}=0.15, \zeta=0.001$, and for different values of $A$

fier chosen. The maximum squeezing of $60 \%$ occurs for $\varepsilon=\kappa=0.3$. The squeezing in general increases with the amplitude of a parametric amplifier for certain values of the cavity damping constant. Despite the fact that it decreases with the cavity damping constant, the cavity light still exhibits the squeezing even for larger values of the cavity damping.

It is not difficult to see from Figs. 2, 3, and 4 that the quadrature variance of the two-mode cavity radiation strongly depends on the linear gain coefficient $A$, amplitude external driving radiation $\zeta$, amplitude of the parametric amplifier $\varepsilon$, mean photon number of the thermal reservoir $\bar{n}_{t h}$, and initial preparation of the atoms $x$.
We now investigate the explicit dependence of the squeezing of the two-mode cavity radiation on these parameters. It is worth noting that the possibility for generating highly squeezed light by altering various parameters will make this system a reliable and attractive source of squeezed light.

We see from Fig. 5 that the degree of squeezing decreases with the mean photon number of the thermal reservoir. We find that there is no restriction on the value of $\bar{n}_{t h}$ for which Eq. (50) has a wellbehaved solution for $x=0.03, A=100, \kappa=0.75$, and $\varepsilon=0.5$. We observe that the squeezing is significantly degraded by the thermal noise. This is due to the influence of the thermal reservoir that decoherences the correlations induced in the two cavity modes by the initial preparation atoms and external driving radiation. A small effect of the reservoir can outweigh to break the correlations between the cavity modes thereby causing the two-mode light to follow the classical properties in which the squeezing cannot be observed. This result is in complete agreement with the result that has been reported for a Correlated Emission Laser (CEL) in [11].

We clearly see from Fig. 6 that the degree of squeezing increases with the linear gain coefficient for $x \leq$ 0.4 and decreases for $x \geq 04$. This indicates that the more atoms are injected into the cavity at a time, the more the degree of the squeezing of the cavity radiation would be. In particular, a maximum squeezing of $66 \%$ occurs for $x=0.02$ and $A=100$. This encouraging result compared with the degree of the squeezing generated by a parametric oscillator in which the maximum possible squeezing is $50 \%$ [26]. Though the squeezing increases with the linear gain coefficient in this case, we cannot use arbitrary values of $A$, since the steady state consideration fails $\left(\lambda_{-}<0\right)$ to be applied for larger values of the linear gain coefficient. Moreover, it is found that a further increment in the linear gain coefficient for which $\lambda_{-} \geq 0$ does not lead to a substantial squeezing.

We clearly see from Fig. 7 that the two-mode cavity radiation exhibits the squeezing for the external driving radiation of a relatively smaller amplitude. The degree of squeezing decreases with the amplitude of the coherent radiation. This indicates the fact that pumping the atoms with stronger radiation than required destroys the squeezing.

Moreover, the effects of the cavity damping constant and linear gain coefficient is indicated in Fig. 8.

ISSN 2071-0194. Ukr. J. Phys. 2021. Vol. 66, No. 3 
It is again observed that the squeezing increases with the linear gain coefficient specially for the smaller cavity damping constant. It is found that, for larger cavity damping constant, a substantial degree of squeezing cannot be achieved no matter how we increase the linear gain coefficient. This is related to a well-known fact that the lesser the cavity damping constant, the more the radiation stays in the cavity which, in turn, enhances the correlation that leads to the squeezing.

It can also easily seen in Fig. 9 that the squeezing increases with the amplitude of a parametric amplifier. It is encouraging that the parametric amplifier can amplify the squeezing in some degree against the loss due the thermal noise. Such property of the parametric amplifier is also observed for a degenerate three-level laser [18] and a nondegenerate three-level laser [20] coupled to the vacuum environment. The squeezing rapidly decays near and at the threshold. The effect of the pumping radiation is seen to improve the degree of squeezing for the smaller values of $\varepsilon$.

\section{Entanglement Amplification}

Here, we proceed to study the entanglement condition of the two modes in the cavity. A pair of particles is taken to be entangled in quantum theory, if its states cannot be expressed as a product of the states of its individual constituents. The preparation and manipulation of these entangled states that have nonclassical and nonlocal properties lead to a better understanding of the basic quantum principles. That is, if the density operator for the combined state cannot be described as a combination of the product of density operators of the constituents,

$\hat{\rho} \neq \sum_{j} P_{j} \hat{\rho}_{j}^{(1)} \otimes \hat{\rho}_{j}^{(2)}$,

where $P_{j} \geq 0$, and $\sum_{j} P_{j}=1$ is set to ensure the normalization of the combined density of state. On the other hand, a maximally entangled continuous variable state can be expressed as a co-eigenstate of a pair of EPR-type operators [24] such as $\hat{X}_{a}-\hat{X}_{b}$ and $\hat{P}_{a}+\hat{P}_{b}$. The total variance of these two operators reduces to zero for maximally entangled continuous variable states. But, according to the criteria set by Duan et al. [25], the quantum states of the system are entangled, if the sum of the variances of a pair of EPR-like operators

$\hat{u}=\hat{X}_{a}-\hat{X}_{b}$,

$\hat{v}=\hat{P}_{a}+\hat{P}_{b}$

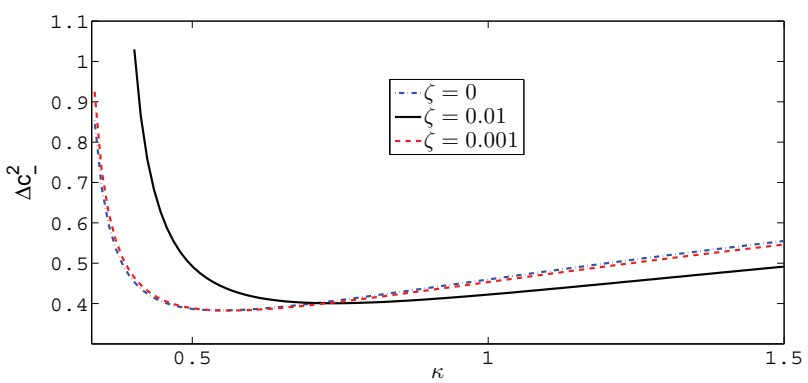

Fig. 7. Plots of the minus quadrature variance [Eq. (50)] of the two-mode cavity radiation at the steady state for $x=0.03$, $A=100, \varepsilon=0.5, \bar{n}_{\mathrm{th}}=0.15$, and for different values of $\zeta$

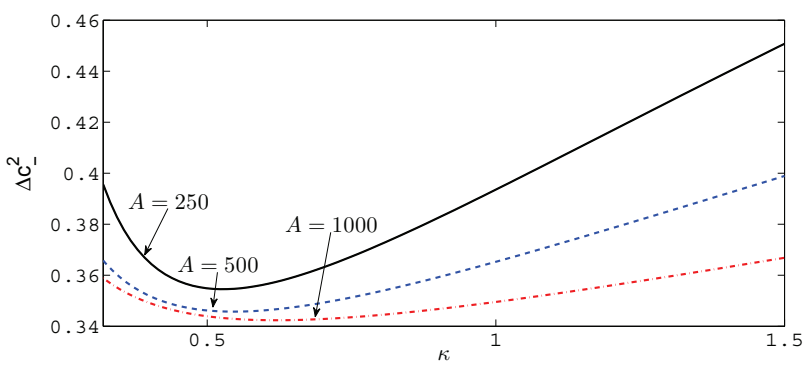

Fig. 8. Plots of the minus quadrature variance [Eq. (50)] of the two-mode cavity radiation at the steady state for $x=0.03$, $\zeta=0.01, \varepsilon=0.5, \bar{n}_{\mathrm{th}}=0.15$, and for different values of $A$

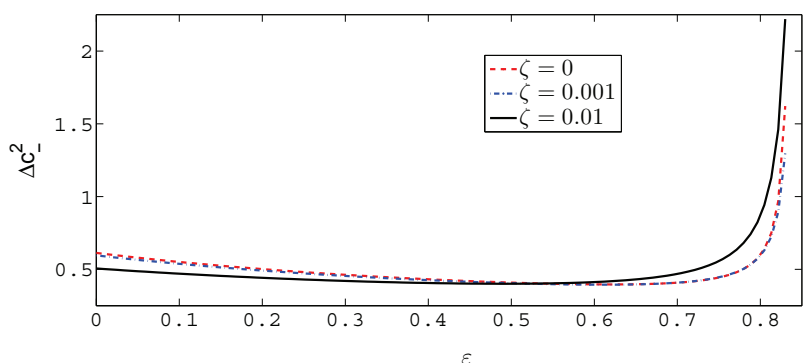

Fig. 9. Plots of the minus quadrature variance [Eq. (50)] of the two-mode cavity radiation at the steady state for $x=0.03$, $\kappa=0.75, \bar{n}_{\text {th }}=0 ., A=100$, and for different values of $\zeta$

where $\hat{X}_{a}=\left(\hat{a}^{\dagger}+\hat{a}\right) / \sqrt{2}, \hat{X}_{b}=\left(\hat{b}^{\dagger}+\hat{b}\right) / \sqrt{2}, \hat{P}_{a}=$ $=i\left(\hat{a}^{\dagger}-\hat{a}\right) / \sqrt{2}, \hat{P}_{b}=i\left(\hat{b}^{\dagger}-\hat{b}\right) / \sqrt{2}$, are quadrature operators for mode $a$ and $b$, satisfy

$\Delta u^{2}+\Delta v^{2}<2$

Thus, the sum of the variances of $\hat{u}$ and $\hat{v}$ is easily found to be

$\Delta u^{2}+\Delta v^{2}=2\left[1+\left\langle\alpha^{*}(t) \alpha(t)\right\rangle+\left\langle\beta^{*}(t) \beta(t)\right\rangle-\right.$

$-2\langle\alpha(t) \beta(t)\rangle]$. 


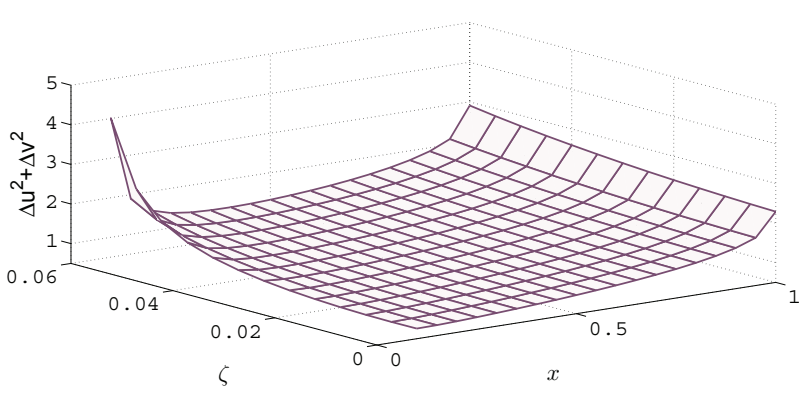

Fig. 10. A plot of the steady-state entanglement [Eq. (62)] of the two-mode cavity radiation for $A=100, \bar{n}_{t h}=0.15$, $\kappa=0.75$, and $\varepsilon=0.5$

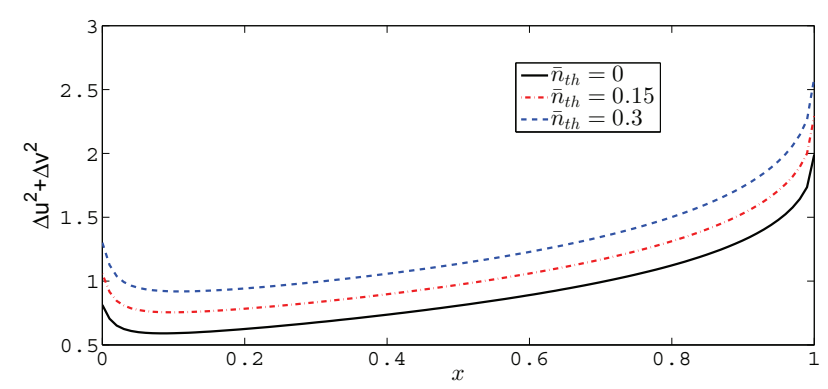

Fig. 11. A plot of the steady-state entanglement [Eq. (62)] of the two-mode cavity radiation for $A=100, \zeta=0.001, \kappa=$ $=0.75, \varepsilon=0.5$, and for different values of $\bar{n}_{\mathrm{th}}$

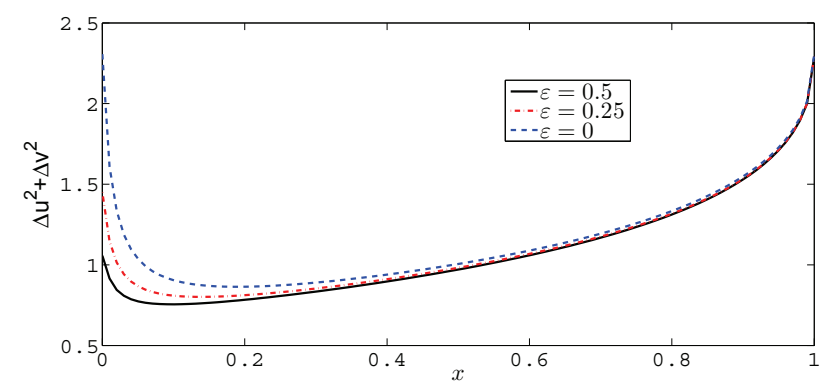

Fig. 12. Plots of the steady-state entanglement [Eq. (62)] of the two-mode cavity radiation for $A=100, \zeta=0.001, \kappa=$ $=0.75, \bar{n}_{\mathrm{th}}=0.15$, and for different values of $\varepsilon$

The term in the parentheses in Eq. (61) exactly represents the minus quadrature variance of the two-mode cavity radiation. We can rewrite Eq. (61) as

$\Delta u^{2}+\Delta v^{2}=2 \Delta c_{-}^{2}$,

where $\Delta c_{-}^{2}$ is given in Eq. (50). It can be noted that the degree of entanglement is directly proportional to the degree of squeezing of the two-mode cavity light.

It is clearly indicated by Fig. 10 that the cavity radiation is entangled for all parameters under consider-

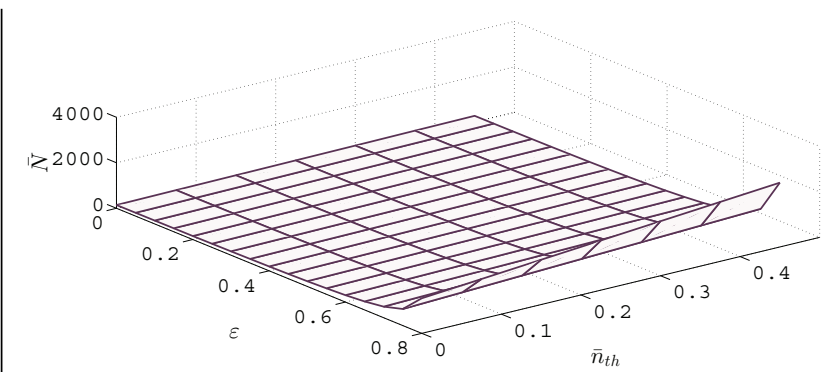

Fig. 13. A plot of the mean number of photon pairs of the cavity radiation (Eq. (65)) at the steady state for $x=0.03$, $\kappa=0.75, \zeta=0.03$, and $A=100$

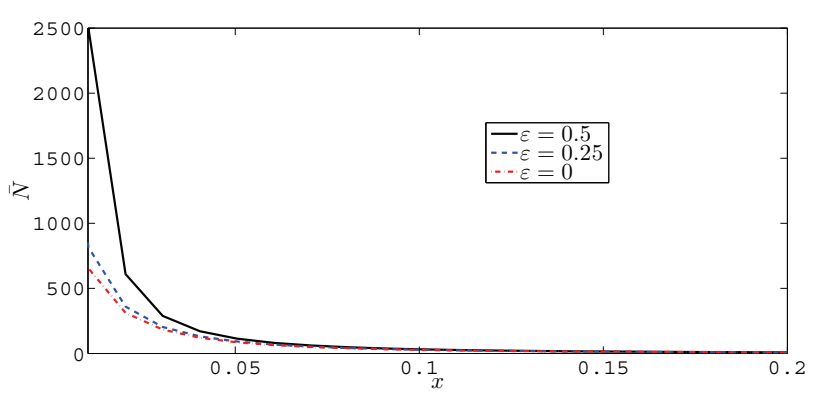

Fig. 14. Plots of the mean number of photon pairs of the cavity radiation (Eq. (65)) at the steady state for $\varepsilon=0.25$, $\kappa=0.75, \zeta=0.001, A=100$, and for different values of $\bar{n}_{t h}$

ation except for the initial atomic coherence $x=1$. It can also be observed that the degree of entanglement increases for smaller values of the initial preparation of atoms and the amplitude of the driving radiation, but decreases for the larger values. Moreover, it can be noticed from Fig. 11 that the entanglement of the cavity radiation decays with the thermal noise and even disappears for larger values of the mean photon number of the thermal environment. The parametric amplifier and the thermal noise have competing effects on the entanglement properties of the cavity radiation.

On the other hand, as is clearly seen in Fig. 12, the degree of entanglement for the quantum system under consideration can be enhanced by increasing the amplitude of a parametric amplifier. It is found that the effect of the parametric amplifier in this case can provide a significant degree of entanglement for smaller values of the linear gain coefficient and the initial preparation of atoms. The effect of a parametric amplifier is insignificant for larger values of the linear gain coefficient, and when a larger number of atoms are initially prepared in the bottom level.

ISSN 2071-0194. Ukr. J. Phys. 2021. Vol. 66, No. 3 


\section{Mean Number of Photon Pairs}

In order to learn about the brightness of the generated light, it is necessary to study the mean number of photon pairs describing the two-mode cavity radiation that can be defined as

$\bar{N}=\left\langle\hat{c}^{\dagger}(t) \hat{c}(t)\right\rangle$

where $\hat{c}(t)$ is the annihilation operator given by Eq. (62). On the basis that the operators in Eq. (63) are already put in the normal order, it is possible to rewrite it in terms of $c$-number variables associated with the normal ordering as

$\bar{N}=\frac{1}{2}\left[\left\langle\alpha^{*}(t) \alpha(t)\right\rangle+\left\langle\beta^{*}(t) \beta(t)\right\rangle\right]$.

Since $\left\langle\alpha^{*}(t) \alpha(t)\right\rangle$ and $\left\langle\beta^{*}(t) \beta(t)\right\rangle$ represent the mean photon numbers in mode $a$ and mode $b$, respectively, $\bar{N}$ can be interpreted as the mean number of photon pairs. It is easy to verify that Eq. (64) represents the mean number of photon pairs $\bar{N}$ of the system. Moreover, with the aid of Eqs. (47) and (48), one can readily verify that

$$
\begin{aligned}
& \left.\bar{N}=\left[\frac{A \Gamma}{2 \Delta}+\kappa \bar{n}_{\mathrm{th}}\right)\right]\left[\frac{\left(\lambda_{+}+\lambda_{-}\right)^{2}+4 \lambda_{+} \lambda_{-}}{8 \lambda_{+} \lambda_{-}\left(\lambda_{+}+\lambda_{-}\right)}\right]+ \\
& +\left[\frac{A \Gamma}{2 \Delta}\left(p^{2}+q_{-}^{2}\right)+\frac{\kappa \bar{n}_{\mathrm{th}}}{2}\left(2 p^{2}+q_{-}^{2}+q_{+}^{2}\right)+\right. \\
& \left.+\left(\frac{A \chi}{2 \Delta}-\varepsilon\right)\left[p\left(q_{+}-q_{-}\right)\right]\right]\left[\frac{\left(\lambda_{+}+\lambda_{-}\right)^{2}-4 \lambda_{+} \lambda_{-}}{8 \lambda_{+} \lambda_{-}\left(\lambda_{+}+\lambda_{-}\right)}\right]+ \\
& +\left[\frac{A \Gamma}{\Delta} p+\left(\frac{A \chi}{2 \Delta}-\varepsilon\right)\left(q_{+}+q_{-}\right)\right]\left[\frac{\left(\lambda_{+}-\lambda_{-}\right)}{8 \lambda_{+} \lambda_{-}}\right] .
\end{aligned}
$$

The result presented in Fig. 13 indicates that the mean number of photon pairs increases with the mean photon number of the thermal reservoir and the amplitude of a parametric amplifier. The effects of the former and latter parameters are also reported, respectively, in Refs. [11] and [19].

Figure 14 explicitly illustrates the dependence of the mean number of photon pairs on the mean number of the thermal reservoir and the initial preparation of atoms. The effect of the thermal reservoir is to increase the mean number of photon pairs which we believe here is the encouraging result. This effect is more prominent nearly at the maximum atomic coherence. Moreover, it is easy to see that the mean

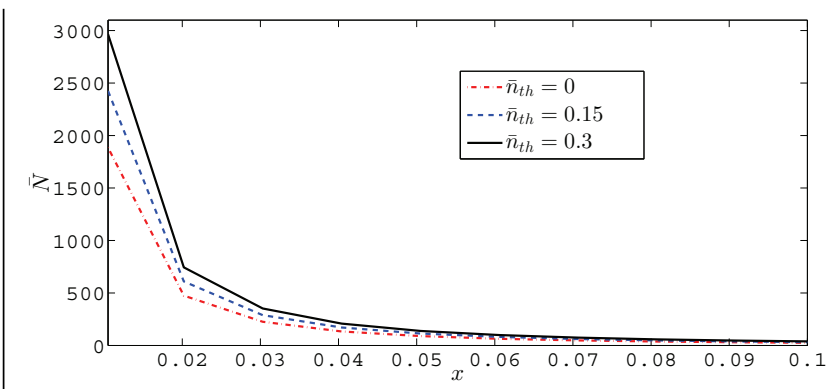

Fig. 15. Plots of the mean number of photon pairs of the cavity radiation (Eq. (65)) at the steady state for $x=0.03$, $\kappa=0.75, \bar{n}_{\text {th }}=0.15, A=100$, and for different values of $\varepsilon$

number of photon pairs appears to be smaller, when more atoms are prepared initially at the bottom level.

Moreover, it is reflected in Fig. 15 that the effect of the parametric amplifier enhances the mean number of photon pairs. Therefore, it worth noting that the careful manipulation of the amplitude of the parametric amplifier and mean photon number of the thermal reservoir can produce an intense light with the considerable entanglement and squeezing.

\section{Conclusion}

We have studied the entanglement and squeezing properties of the two-mode light generated by a nondegenerate three-level laser with a nondegenerate parametric amplifier and coupled with the thermal reservoir. We have obtained the master equation in the good-cavity limit and in the linear and adiabatic approximation schemes. Applying the master equation, we have derived equations of evolution of the moments of the cavity mode variables. Making use of these equations, we have calculated the quadrature variance for the two-mode light inside the cavity at the steady state. We have also analyzed the entanglement of the two mode cavity light at the steady state. Finally, we have calculated the mean photon number for the two-mode cavity light.

It is found that the effect of the parametric amplifier in the laser cavity to produce a robust squeezed and entangled light that remains stronger even for the larger values of the cavity damping constant. Moreover, the parametric amplifier enhances the degree of squeezing and entanglement when more atoms are initially prepared at the top level, and its effect in increasing the squeezing is limited for very small amplitude of the external driving radiation. Moreover, 
we find the mean number of photon pairs increase with the mean photon number of the thermal reservoir and the parametric amplifier. The mean number of photon pairs decreases with the initial preparation of atoms in which more atoms are found in the ground state and decreases with the amplitude of an external driving radiation as always the case.

\section{APPENDIX A}

\section{Stochastic differential equations}

We now obtain the stochastic differential equations associated with the normal ordering for the cavity mode variables. To this end, we apply Eq. (12) and the fact that

$\frac{d}{d t}\langle\hat{A}\rangle=\operatorname{Tr}\left(\frac{d \hat{\rho}(t)}{d t} \hat{A}\right)$.

The time evolution of the expectation value of the cavity mode variables applying the cyclic property of the trace operation and taking the bosonic commutation relation into account turns out to be

$\frac{d}{d t}\langle\hat{a}\rangle=-\frac{\mu_{a}}{2}\langle\hat{a}\rangle+\frac{\nu_{a}}{2}\left\langle\hat{b}^{\dagger}\right\rangle$,

$\frac{d}{d t}\langle\hat{b}\rangle=-\frac{\mu_{b}}{2}\langle\hat{b}\rangle-\frac{\nu_{b}}{2}\left\langle\hat{a}^{\dagger}\right\rangle$,

$\frac{d}{d t}\left\langle\hat{a}^{2}\right\rangle=-\mu_{a}\left\langle\hat{a}^{2}\right\rangle+\nu_{a}\left\langle\hat{b}^{\dagger} \hat{a}\right\rangle$,

$\frac{d}{d t}\left\langle\hat{b}^{2}\right\rangle=-\mu_{b}\left\langle\hat{b}^{2}\right\rangle-\nu_{b}\left\langle\hat{a}^{\dagger} \hat{b}\right\rangle$,

$\frac{d}{d t}\left\langle\hat{a}^{\dagger} \hat{a}\right\rangle=-\mu_{a}\left\langle\hat{a}^{\dagger} \hat{a}\right\rangle+\frac{\nu_{a}}{2}\left[\left\langle\hat{a}^{\dagger} \hat{b}^{\dagger}\right\rangle+\langle\hat{a} \hat{b}\rangle\right]+$

$+\frac{A \Sigma_{1}}{2 \Delta}+\kappa \bar{n}_{\mathrm{th}}$,

$\frac{d}{d t}\left\langle\hat{b}^{\dagger} \hat{b}\right\rangle=-\mu_{b}\left\langle\hat{b}^{\dagger} \hat{b}\right\rangle-\frac{\nu_{b}}{2}\left[\left\langle\hat{b}^{\dagger} \hat{a}^{\dagger}\right\rangle+\langle\hat{a} \hat{b}\rangle\right]+\kappa \bar{n}_{\mathrm{th}}$,

$\frac{d}{d t}\left\langle\hat{a}^{\dagger} \hat{b}\right\rangle=-\frac{1}{2}\left(\mu_{a}+\mu_{b}\right)\left\langle\hat{a}^{\dagger} \hat{b}\right\rangle-\frac{\nu_{b}}{2}\left\langle\hat{a}^{\dagger 2}\right\rangle+\frac{\nu_{a}}{2}\left\langle\hat{b}^{\dagger 2}\right\rangle$,

$\frac{d}{d t}\langle\hat{a} \hat{b}\rangle=-\frac{1}{2}\left(\mu_{a}+\mu_{b}\right)\langle\hat{a} \hat{b}\rangle-\frac{\nu_{b}}{2}\left\langle\hat{a}^{\dagger} \hat{a}\right\rangle+$

$+\frac{\nu_{a}}{2}\left\langle\hat{b}^{\dagger} \hat{b}\right\rangle-\frac{\nu_{b}}{2}$,

in which

$\mu_{a}=\kappa-\frac{A \Sigma_{1}}{\Delta}, \quad \mu_{b}=\kappa+\frac{A \Sigma_{2}}{\Delta}$,

$\nu_{a}=2 \varepsilon+\frac{A \Sigma_{3}}{\Delta}, \quad \nu_{b}=\frac{A \Sigma_{4}}{\Delta}-2 \varepsilon$.

We note that the operators in the above equations are in the normal order. The $c$-number equations corresponding to Eqs. (A2)-(A9) are

$\frac{d}{d t}\langle\alpha\rangle=-\frac{\mu_{a}}{2}\langle\alpha\rangle+\frac{\nu_{a}}{2}\left\langle\beta^{*}\right\rangle$,

$\frac{d}{d t}\langle\beta\rangle=-\frac{\mu_{b}}{2}\langle\beta\rangle-\frac{\nu_{b}}{2}\left\langle\alpha^{*}\right\rangle$, $\frac{d}{d t}\left\langle\alpha^{2}\right\rangle=-\mu_{a}\left\langle\alpha^{2}\right\rangle+\nu_{a}\left\langle\beta^{*} \alpha\right\rangle$,

$\frac{d}{d t}\left\langle\beta^{2}\right\rangle=-\mu_{b}\left\langle\beta^{2}\right\rangle-\nu_{b}\left\langle\alpha^{*} \beta\right\rangle$,

$\frac{d}{d t}\left\langle\alpha^{*} \alpha\right\rangle=-\mu_{a}\left\langle\alpha^{*} \alpha\right\rangle+\frac{\nu_{a}}{2}\left[\left\langle\alpha^{*} \beta^{*}\right\rangle+\langle\alpha \beta\rangle\right]+$

$+\frac{A \Sigma_{1}}{2 \Delta}+\kappa \bar{n}_{\mathrm{th}}$

$\frac{d}{d t}\left\langle\beta^{*} \beta\right\rangle=-\mu_{b}\left\langle\beta^{*} \beta\right\rangle-\frac{\nu_{b}}{2}\left[\left\langle\beta^{*} \alpha^{*}\right\rangle+\langle\alpha \beta\rangle\right]+\kappa \bar{n}_{\mathrm{th}}$,

$\frac{d}{d t}\left\langle\alpha^{*} \beta\right\rangle=-\frac{1}{2}\left(\mu_{a}+\mu_{b}\right)\left\langle\alpha^{*} \beta\right\rangle-\frac{\nu_{b}}{2}\left\langle\alpha^{* 2}\right\rangle+\frac{\nu_{a}}{2}\left\langle\beta^{* 2}\right\rangle$,

$\frac{d}{d t}\langle\alpha \beta\rangle=-\frac{1}{2}\left(\mu_{a}+\mu_{b}\right)\langle\alpha \beta\rangle-\frac{\nu_{b}}{2}\left\langle\alpha^{*} \alpha\right\rangle+$

$+\frac{\nu_{a}}{2}\left\langle\beta^{*} \beta\right\rangle-\frac{\nu_{b}}{2}$.

1. C.W. Gardiner. Inhibition of atomic phase decays by squeezed light: A direct effect of squeezing. Phys. Rev. Lett. 56, 1917 (1986).

2. Q. Sajid, Q. Shahid, M.S. Zubairy. Effect of phase fluctuations on entanglement generation in a correlated emission laser with injected coherence. Opt. Commun. 283, 781 (2010).

3. E. Alebachew. Enhanced squeezing and entanglement in a non-degenerate three-level cascade laser with injected squeezed light. Opt. Commun. 280, 133 (2007).

4. S. Tesfa. Effect of dephasing on quantum features of the cavity radiation of an externally pumped correlated emission laser. Phys. Rev. A 79, 063815 (2009).

5. N.A. Ansari, J.G. Banacloche, M.S. Zubairy. Phase-sensitive amplification in a three-level atomic system. Phys. Rev. A 41, 5179 (1990).

6. H. Xiong, M.O. Scully, M.S. Zubairy. Correlated spontaneous emission laser as an entanglement amplifier. Phys. Rev. Lett. 94, 023601 (2005).

7. T. Abebe, N. Gemechu. Two-level atom with squeezed light from optical parametric oscillators. Ukr. J. Phys. 63 (7), 600 (2018).

8. S. Qamar, M. Al-Amri, M.S. Zubairy. Entanglement in a bright light source via Raman-driven coherence. Phys. Rev. A 79, 013831 (2009).

9. Ch. Gashu, T. Abebe. Externally induced entanglement amplification in a coherently pumped emission of laser with parametric amplifier and coupled to squeezed vacuum reservoir. Phys. Scr. 95, 075105 (2020).

10. J. Anwar, M.S. Zubairy. Quantum-statistical properties of noise in a phase-sensitive linear amplifier. Phys. Rev. A 49, 481 (1994).

11. S. Tesfa. Effects of decoherence on entanglement in a correlated emission laser. J. Phys. B: At. Mol. Opt. Phys. 40, 2373 (2007).

12. C.A. Blockley, D.F. Walls. Intensity fluctuations in a frequency down-conversion process with three-level atoms. Phys. Rev. A 43 (9), 5049 (1991).

ISSN 2071-0194. Ukr. J. Phys. 2021. Vol. 66, No. 3 
13. N. Lu, F.X. Zhao, J. Bergou. Nonlinear theory of a twophoton correlated-spontaneous-emission laser: A coherently pumped two-level-two-photon laser. Phys. Rev. A 39, 5189 (1989).

14. T. Abebe. The quantum analysis of a nondegenerate threelevel laser with spontaneous emission and noiseless vacuum reservoir. Ukr. J. Phys. 63 (11), 969 (2018).

15. K. Fesseha. Fundamentals of Quantum Optics (Lulu, 2008).

16. N. Geoffery. Introduction to Nonlinear Optics (Cambridge Univ. Press, 2011).

17. W.H. Louisell. Quantum Statistical Properties of Radiation (Wiley, 1973).

18. E. Alebachew, K. Fesseha. A degenerate three-level laser with a parametric amplifier. Opt. Commun. 265, 314 (2006).

19. T.Y. Darge, K. Fesseha. Coherently driven degenerate three-level laser with parametric amplifier. PMC Physics B. 3, 1 (2010).

20. T. Abebe. Enhancement of squeezing and entanglement in a non-degenerate three-level cascade laser with coherently driven cavity. Ukr. J. Phys. 63 (8), 733 (2018).

21. T. Abebe, Ch. Gashu. Dynamics of a nondegenerate threelevel laser with parametric amplifier and coupled to a twomode squeezed vacuum reservoir. Braz. J. Phys. 50 (5), 495 (2020).

22. T. Abebe, N. Gemechu, K. Shogile, S. Hailemariam, Ch. Gashu, Sh. Adisu. Entanglement quantification using various inseparability criteria for correlated photons. Rom. J. Phys. 65, 107 (2020).

23. S. Tesfa. Continuous variable entanglement in a coherently pumped correlated emission laser. J. Phys. B: At. Mol. Opt. Phys. 41, 055503 (2008).

24. A. Einstein, B. Podolsky, R. Rosen. Can quantummechanical description of physical reality be considered complete? Phys. Rev. 47, 777 (1935).
25. L.M. Duan, G. Giedke, J.I. Cirac, P. Zoller. Inseparability criterion for continuous variable systems. Phys. Rev. Lett. 84, 2722 (2000).

26. B. Daniel, K. Fesseha. The propagator formulation of the degenerate parametric oscillator. Opt. Commun. 151, (1998) 384 .

Received 25.11.19

\section{T. Абебе, К. Гашу, E. Mocica}

\section{ГЕНЕРУВАННЯ ІНТЕНСИВНОГО}

ЗАПЛУТАНОГО ПРОМЕНЯ СВІТЛА

НЕВИРОДЖЕНИМ ТРИРІВНЕВИМ ЛАЗЕРОМ

З ПАРАМЕТРИЧНИМ ПІДСИЛЮВАЧЕМ

\section{І ТЕПЛОВИМ РЕЗЕРВУАРОМ}

Дано детальний аналіз двомодового квадратурного стиснення і статистичних властивостей світла, що генерується невиродженим трирівневим лазером з параметричним підсилювачем і тепловим резервуаром. На основі керуючого i стохастичного диференційного рівнянь досліджуються некласичні характеристики світла, генерованого квантовою системою. За допомогою їх розв'язків і кореляційних властивостей операторів шуму знайдено квадратурне стиснення, заплутування і середнє число пар фотонів світла резонатора. Показано, що зовнішнє збуджувальне випромінювання малої амплітуди індукує сильну кореляцію верхнього і нижнього рівнів трирівневих атомів і сильне стиснення. Наявність параметричного підсилювача підвищує ступінь стиснення світла резонатора. Встановлено, що зростання середнього числа теплових фотонів зменшує стиснення, але збільшує середнє число пар фотонів світла резонатора.

Ключовi cлова: параметричний підсилювач, квадратурне стиснення, заплутування, середнє число пар фотонів 